\section{ORIGINAL RESEARCH}
N. Bergsland
D. Horakova
M.G. Dwyer
O. Dolezal
Z.K. Seidl
M. Vaneckova
J. Krasensky
E. Havrdova
R. Zivadinov

\title{
Subcortical and Cortical Gray Matter Atrophy in a Large Sample of Patients with Clinically Isolated Syndrome and Early Relapsing-Remitting Multiple Sclerosis
}

BACKGROUND AND PURPOSE: Recent studies have shown that selective regional, but not global, GM atrophy occurs from clinical onset to conversion to clinically definite MS. Our aim was to investigate the difference in the extent of SDGM and cortical atrophy in a large sample of patients with $\mathrm{CIS}$ and early RRMS and to explore the relationship between SDGM and cortical atrophy and other MR imaging and clinical outcomes.

MATERIALS AND METHODS: Two hundred twelve patients with CIS recruited the first clinical event (mean age, 29.3 years; median EDSS, 1.5; median disease duration, 3 months) and 177 patients with early RRMS (mean age, 30.7 years; median EDSS, 2.0; median disease duration, 47 months) were imaged on a 1.5T scanner by using a high-resolution 3D T1 spoiled gradient-recalled sequence. Volumetric data for SDGM structures were obtained by using FSL FIRST, while whole-brain, GM, white matter, cortical, and lateral ventricle volumes were estimated by using SIENAX software. Comparisons between the groups were adjusted for age and sex.

RESULTS: Patients with early RRMS showed significantly lower SDGM but not cortical volumes compared with patients with CIS. The most apparent SDGM differences were evident in the caudate and thalamus $(P<.0001)$, total SDGM $(P=.0001)$, and globus pallidus $(P=.01)$. Patients with $\mathrm{CIS}$ with a median T2 lesion volume $>4.49 \mathrm{~mL}$ showed lower total SDGM, caudate, thalamus $(P<.001)$, globus pallidus $(P=.007)$, hippocampus $(P=.004)$, and putamen $(P=.01)$ volumes and higher lateral ventricle volume $(P=.001)$ than those with a median T2 lesion volume $<4.49 \mathrm{~mL}$. Decreased thalamic volume showed the most consistent relationship with MR imaging outcomes $(P<.0001)$ in patients with $\mathrm{CIS}$.

CONCLUSIONS: Significant SDGM, but not cortical, atrophy develops during the first 4 years of the RRMS. GM atrophy is relevant for disease progression from the earliest clinical stages.

ABBREVIATIONS: ASA = Avonex-Steroid-Azathioprine; $\mathrm{CIS}=$ clinically isolated syndrome; $\mathrm{EDSS}=$ Expanded Disease Status Scale; FSL = FMRIB Software Library; GM = gray matter; NBV = normalized brain volume; NCV = normalized cortical volume; NGMV = normalized gray matter volume; NLVV = normalized lateral ventricle volume; NWMV = normalized white matter volume; RRMS = relapsing remitting MS; SDGM = subcortical deep gray matter; SET = Study of Early Interferon $\beta$ 1a Treatment in High Risk Subjects after CIS

M $\mathrm{R}$ imaging is a vital tool enabling clinicians to diagnose, monitor, and predict the progression of MS. Conventional MR imaging has proved to be very sensitive for detecting focal changes in the WM, yet it is relatively insensitive to involvement of the GM in MS. GM pathology in MS is quite different from that in WM, with only mild blood-brain barrier disruption and little-to-no inflammation due to minimal Tcell infiltration. ${ }^{1}$ Hence, the difference between lesion and

Received August 12, 2011; accepted after revision November 18.

From the Buffalo Neuroimaging Analysis Center (N.B., M.G.D., O.D., R.Z.) and Jacobs Neurological Institute (R.Z.), University at Buffalo, Buffalo, New York; and Department of Neurology and Center of Clinical Neuroscience (D.H., O.D., E.H.) and Department of Radiology (Z.K.S., M.V., J.K.), Charles University in Prague, First Faculty of Medicine, Prague, Czech Republic.

Please address correspondence to Robert Zivadinov, MD, PhD, FAAN, Department of Neurology, School of Medicine and Biomedical Sciences, Buffalo Neuroimaging Analysis Center, 100 High St, Buffalo, NY 14203; e-mail: rzivadinov@bnac.net

Indicates open access to non-subscribers at www.ajnr.org

Indicates article with supplemental on-line table.

http://dx.doi.org/10.3174/ajnr.A3086 normal GM relaxation times on MR imaging is less than that seen between lesion and normal white matter. ${ }^{2}$

In the past decade, continuous effort has been made to develop novel MR imaging techniques that are able to quantitatively measure a wide spectrum of GM pathology. ${ }^{1-3}$ The introduction of double inversion recovery sequences has advanced the ability to capture part of GM pathology in vivo, ${ }^{4}$ and measurement of GM atrophy has become one of the important outcomes in MS studies. ${ }^{5-7}$ Other nonconventional MR imaging techniques have also contributed to determining the extent of GM damage in patients with MS. ${ }^{2}$ The role of GM pathology in MS has come under increasingly close scrutiny, especially after it was shown that GM atrophy predicts clinical outcomes better than WM damage. ${ }^{5-7}$

More recently, research has focused on determining the extent of GM pathology at the first clinical event in patients presenting with $\mathrm{CIS}^{8-16}$ or on its evolution with conversion to clinically definite MS. ${ }^{17-20}$ It has been reported that global GM volume measures are not sensitive enough to detect GM atrophy at the time of the initial attack. ${ }^{11}$ Consequently, GM atrophy studies in patients with CIS have 
increasingly turned to regional segmentation techniques to identify specific structures with a stronger predilection for disease susceptibility and conversion to clinically definite MS. ${ }^{8-11,13,14,16,17,19,21}$

The aim of this study was to investigate differences in the extent of SDGM and cortical atrophy in a large sample of patients with CIS and early RRMS. Another goal was to explore the relationship between SDGM and cortical atrophy and other MR imaging and clinical outcomes in these disease subtypes.

\section{Materials and Methods}

\section{Subjects}

The current analysis is based on MR imaging baseline data from 2 multicenter longitudinal clinical trials conducted in the Czech Republic. $^{22,23}$ The randomized double-blind placebo-controlled ASA study enrolled 181 patients with early RRMS, ${ }^{22}$ while the observational SET study enrolled 220 patients with CIS. ${ }^{23}$ The inclusion criteria for the ASA study were clinically definite MS according to the criteria of Poser et $\mathrm{al}^{24}$ confirmed by MR imaging, the presence of at least 2 oligoclonal bands in the CSF, $18-55$ years of age, EDSS score $\leq 3.5$ on the day of screening, and active disease defined by 2 relapses in the previous 12 months or 3 relapses in the previous 24 months. ${ }^{22}$ Exclusion criteria were interferon- $\beta$ therapy in the previous 12 months or immunosuppressive treatment in the previous 6 months, pregnancy, and active major organ disease. All enrolled patients were naïve to interferon- $\beta$ and glatiramer acetate at study entry. ${ }^{22}$

Inclusion criteria for the SET study were $18-55$ years of age, EDSS score of $\leq 3.5$ on day of screening, presence of $\geq 2$ T2 hyperintense lesions on diagnostic MR imaging, and the presence of $\geq 2$ oligoclonal bands in the CSF (both obtained before steroid treatment). Exclusion criteria were time from the beginning of first symptoms of CIS to the baseline visit no longer than 4 months and the occurrence of a second attack before the baseline visit. All patients were treated with 3-5 g of methylprednisolone for the first symptoms, and baseline MR imaging was performed at least 30 days after steroid administration.

All participants provided written informed consent to participate in the study that was approved by the local institutional review boards.

\section{MR Imaging Methods}

Image Acquisition. Subjects in both studies were scanned on the same Gyroscan 1.5T unit (Philips Healthcare, Best, the Netherlands). Axial images of the brain were acquired with $1.5-\mathrm{mm}$ section thickness by using FLAIR spin-echo (TR/TE/TI, 11,000/140/2600 ms; matrix size, $256 \times 181 \mathrm{~mm}$; flip angle, $\left.90^{\circ}\right)$ and axial T1-weighted 3D spoiled gradient-recalled images (TR/TE, $25 / 5 \mathrm{~ms}$; matrix size, $256 \times$ $204 \mathrm{~mm}$; flip angle, $30^{\circ}$ ) acquired with 1-mm section thickness; sections were contiguous. The SET study also included a T1 spin-echo postcontrast sequence (TR/TE, 500/11 ms; matrix size, $256 \times 180$ $\mathrm{mm}$; flip angle, $90^{\circ}$; 3-mm section thickness) acquired with a 5-minute delay after the administration of gadolinium diethylene-triamine pentaacetic acid.

Image Analysis. All image analyses were conducted blinded to disease status. T2 and gadolinium lesion volumes were calculated for both the CIS and RRMS groups by using a reliable contouring-thresholding technique, as previously described. ${ }^{22,23,25} \mathrm{~T} 2$ and gadolinium lesion numbers were also obtained in the CIS group only.

Before we used the T1-weighted 3D spoiled gradient-recalled for subsequent analysis, it was modified by using an in-house-developed in-painting technique to avoid the impact of WM lesions on GM volume measurements ${ }^{26}$ as previously described. ${ }^{27}$ Consequently, for each subject, we obtained NBV, NGMV, NCV, NWMV, NLVV, and a volumetric scaling factor, by using SIENAX (Version 2.6; FMRIB Software Library, http://www.fmrib.ox.ac.uk/fsl/). ${ }^{27}$ Absolute tissue volumes for the thalamus, caudate, putamen, globus pallidus, hippocampus, amygdala, and nucleus accumbens were estimated from the in-painted T1-weighted 3D spoiled gradient-recalled with FIRST (Version 1.2, FMRIB Software Library), a model-based segmentation/registration tool. ${ }^{28}$ Normalized SDGM volumes were thus obtained by multiplying the estimated volumes from FIRST by the volumetric scaling factor from SIENAX. ${ }^{29}$ Reproducibility of FIRST was previously reported. ${ }^{29}$

Statistical Analysis. Statistical analyses were performed by using the Statistical Package for the Social Sciences for Windows (Version 16; SPSS, Chicago, Illinois). Data were first examined visually and statistically by using the Kolmogorov-Smirnov test for normality. Demographic, clinical, and MR imaging differences between the groups were tested by using the $\chi^{2}$ test, Student $t$ test, and Mann-Whitney rank sum test, as appropriate.

We investigated whether there were any laterality differences in the SDGM structures. Because the left and right volumes for each structure were highly correlated (data not shown), they were combined to yield a single structural volume. Only the total volumes for each structure were kept in the statistical models to reduce the total number of comparisons. Mean regional GM volumetric differences between the study groups were investigated before and after correction for age and sex, by using the Student $t$ test and analysis of covariance.

To investigate the differences in global and regional brain volumes, we further divided patients with CIS into subgroups according to T2 lesion volume (median, $>4.49 \mathrm{~mL}$ or $<4.49 \mathrm{~mL}$ ), T2 lesion number ( $\geq 9$ and $<9$ ), gadolinium-positivity status, polysymptomatic-versus-monosymptomatic onset, and different subtypes of monosymptomatic onset.

Spearman rank correlation was used to assess the relationships between regional GM volumes and other MR imaging and clinical outcomes in the CIS and RRMS groups. Due to multiple comparisons, only nominal $P$ values $<.01$ were considered statistically significant by using 2 -tailed tests.

\section{Results}

\section{Demographic, Clinical, and MR Imaging Characteristics}

Two-hundred twelve patients with CIS and 177 patients with early relapsing-remitting MS were enrolled in this study. SDGM analysis was not possible for 8 patients with CIS and 4 with early RRMS who originally participated in the SET and ASA studies due to technical difficulties. No significant demographic, clinical, or MR imaging differences were found between the subjects enrolled in this study and those participating in the original clinical trials..$^{22,23}$

Demographic and clinical characteristics of the study groups are shown in the On-line Table. There were no significant age differences between the study groups; however, there was a trend toward more female patients with early RRMS $(P=.013)$. As expected, patients with early RRMS had significantly longer median disease duration (47 versus 3 months, $P<.0001)$ and a trend toward higher median EDSS (2.0 versus 
Table 1: Volumetric regional gray matter differences in patients with clinically isolated syndrome compared with patients with early relapsing-remitting multiple sclerosis ${ }^{a}$

\begin{tabular}{|c|c|c|c|c|c|c|}
\hline & \multirow{2}{*}{$\begin{array}{l}\text { CIS }(n=212) \\
\text { (mean) (SD) }\end{array}$} & \multirow{2}{*}{$\begin{array}{c}\text { Early RRMS ( } n=177) \\
\text { (mean) (SD) }\end{array}$} & \multicolumn{4}{|c|}{ Volumetric Differences between CIS and Early RRMS ${ }^{\mathrm{b}}$} \\
\hline & & & $\%$ Difference & SED & $P$ Value & Adjusted $P$ Value \\
\hline NCV & $614.24(33.11)$ & $609.41(38.69)$ & -0.79 & 3.64 & .186 & .532 \\
\hline Total SDGMV & $63.24(3.93)$ & $61.9(5.12)$ & -2.12 & 0.46 & .004 & .001 \\
\hline Caudate & $9.33(1.00)$ & $8.96(1.03)$ & -3.97 & 0.1 & $<.0001$ & $<.0001$ \\
\hline Putamen & $13.29(1.04)$ & $13.04(1.32)$ & -1.88 & 0.12 & .040 & .051 \\
\hline Globus pallidus & $4.74(0.36)$ & $4.66(0.93)$ & -1.69 & 0.04 & .022 & .01 \\
\hline Thalamus & $21.25(1.50)$ & $20.42(1.97)$ & -3.91 & 0.18 & $<.0001$ & $<.0001$ \\
\hline Hippocampus & $9.83(0.94)$ & $10.02(1.10)$ & 1.93 & 0.1 & .068 & .251 \\
\hline Amygdala & $3.52(0.44)$ & $3.56(0.42)$ & 1.14 & 0.04 & .416 & .523 \\
\hline Nucleus accumbens & $1.3(0.24)$ & $1.27(0.26)$ & -2.31 & 0.03 & .228 & .213 \\
\hline
\end{tabular}

Note:-SDGMV indicates subcortical deep gray matter volume; SED, standard error of difference.

Note:-
all volumes are reported in milliliters.

${ }^{b}$ Student $t$ test and analysis of variance were used to assess volumetric differences between CIS and RRMS groups.

$1.5, P=.035)$ compared with patients with CIS. Ninety-four $(44.3 \%)$ of the patients with CIS presented with polysymptomatic onset, and 118 (55.7\%), with monosymptomatic onset (On-line Table).

The mean number of T2 lesions in patients with CIS was $13.4 \pm 8.5$, and $63.2 \%$ of patients with CIS had $>9$ hyperintense T2 lesions. Patients with early RRMS showed significantly higher T2 lesion volume compared with the patients with CIS $(11.3 \mathrm{~mL}$ versus $6.2 \mathrm{~mL}, P<.0001)$. Patients with early RRMS showed significantly higher NLVV compared with patients with CIS $(P=.002)$, but no significant differences were found between the 2 groups for NBV, NGMV, and NWMV.

\section{Subcortical Regional Gray Matter Volume Differences between Patients with CIS and those with RRMS}

Table 1 and Fig 1 show the volumetric differences in patients with CIS compared with those with early RRMS. The data are provided with and without adjustment for age and sex. Patients with early RRMS showed significantly lower volumes in the caudate and thalamus $(P<.0001)$, total SDGM $(P=$ $.0001)$, and globus pallidus $(P=.01)$ compared with patients with CIS. The 2 groups did not differ significantly in NCV.

\section{Global and Regional Volume Differences in the Patients with CIS}

Patients with CIS with T2 lesion volume greater than the median $(4.49 \mathrm{~mL})$ showed lower total SDGM, caudate, thalamus (all $P<.001)$, globus pallidus $(P=.007)$, hippocampus $(P=$ $.004)$, and putamen $(P=.01)$ volumes and higher NLVV $(P=$ .001) than those with a median T2 lesion volume $<4.49 \mathrm{~mL}$ (Table 2). The 2 groups did not differ significantly in NCV. No age or sex differences were found between the 2 subgroups, whereas patients with CIS with median T2 lesion volume $<4.49 \mathrm{~mL}$ had a slightly longer disease duration (1.2 months, $P=.005)$.

No significant differences for the brain volume measures were found between the CIS subgroups when divided for the number of T2 lesions, gadolinium-positivity status, polysymptomatic-versus-monosymptomatic onset, and subtypes of monosymptomatic onset. However, patients with polysymptomatic onset showed significantly higher gadolinium lesion volume $(P=.009)$ and T2 lesion volume $(P=.003)$ and a trend toward higher gadolinium $(P=.021)$ and T2 $(P=$ .038) lesion numbers.

\section{Relationship between Regional Gray Matter Volumes and Other MR Imaging and Clinical Outcomes in Patients with CIS and Early RRMS}

Correlations between regional GM volumes and other MR imaging and clinical outcomes in patients with CIS and those with early RRMS are presented in Table 3.

In patients with CIS, significant relationships between the decreased total SDGM and increased T2 lesion volume and NLVV, and decreased NBV were found (<.0001). Decreased volume of the thalamus showed the most consistent relationship with MR imaging outcomes (all, $P<.0001)$. No relationship was found between regional GM volumes and disability. Except for the amygdala, no relationship was found with disease duration.

Modest-to-strong relationships were detected in patients with early RRMS between most of the GM regions and MR imaging outcomes. The most robust correlations were detected for total SDGM and thalamus $(P<.0001)$. Decreased volumes of the thalamus and NCV were the only regions that were significantly related to increased $\operatorname{EDSS}(P=.007$ and $P=$ .006 , respectively), while total SDGM and caudate volumes showed a trend toward a relationship with EDSS. Longer disease duration was related to decreased NCV; total SDGM; and caudate, putamen, and thalamus volumes (all $P<.0001$ ).

\section{Discussion}

This is the largest study to date, to our knowledge, to investigate the extent of SDGM and cortical atrophy in patients with CIS and those with early RRMS. The data for this study were acquired with the same scanning protocol and on the same MR imaging scanner, as part of the ongoing SET and ASA clinical trials in MS. This process allowed us to directly compare the evolution of SDGM and cortical atrophy between patients with CIS and those with early RRMS with only 4 years of disease duration.

The early RRMS group showed significant decreases in multiple SDGM regions compared with the patients with CIS, and these were most evident in total SDGM, caudate, thalamus, and globus pallidus. Although a number of previous studies evaluated the extent of SDGM atrophy in 


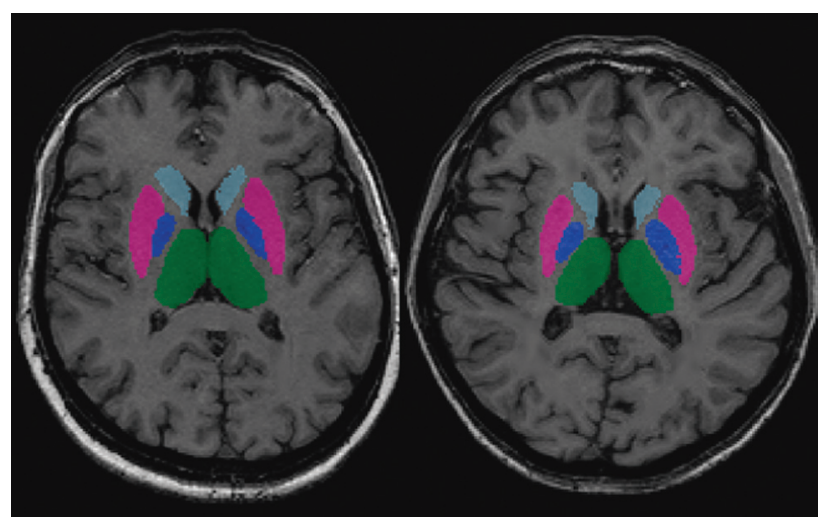

Fig 1. Representative FIRST segmentation in a 47-year-old female patient with CIS (EDSS 1.5) on the left and a 47-year-old female patient with RRMS (EDSS 2.0) on the right. The colored regions represent thalamus (green), globus pallidus (dark blue), caudate (light blue), and putamen (magenta).

RRMS, $8,11,16,30,31$ the present study provides evidence that changes in SDGM occur rapidly during the first 4 years in patients untreated with currently FDA-approved diseasemodifying treatment. In fact, all enrolled patients were naïve to interferon- $\beta$ and glatiramer acetate at entry into the ASA study. ${ }^{22}$ No significant differences were found in whole-brain, global GM, WM, and/or cortical volumes between the 2 groups. This confirms the results of some recent studies that showed that selective regional, but not global, atrophy occurs from clinical onset to conversion to clinically definite MS. ${ }^{17-20}$ This study provides strong evidence that regional GM atrophy is exclusively confined to the SDGM and not to cortical regions in the first 4 years of disease duration. Our findings suggest, therefore, that future studies should concentrate more on understanding the pathogenetic mechanisms leading to selective SDGM rather than to cortical GM damage in patients with early RRMS. Central atrophy development in early RRMS (10\% larger NLVV than that in patients with CIS) emphasizes the need for better understanding of pathogenetic mechanisms leading to selective central and SDGM atrophy.

The disparate findings of GM atrophy in patients with CIS between different studies ${ }^{7-9,11,14,16,21}$ point to the heterogeneity of the disease in patients with CIS and the inclusion criteria for the studies in which they are enrolled. A recent study revealed significant global GM volumetric differences in contrast to our own between patients with CIS and RRMS ${ }^{16}$; how- ever, patients with CIS did not have to fulfill the oligoclonal bands criterion. It could be postulated that the more restrictive enrollment criteria used in the present study selected a cohort of patients more prone to convert to clinically definite MS. It remains difficult to directly compare the results between studies. Despite this important caveat, the present study, with its large sample size, supports the hypothesis that pathogenic processes affect both GM and WM from the earliest stages of the disease.

The decision to divide the CIS group into those with $\geq 9$ or $<9$ T2 lesions and according to gadolinium-positivity status was based on the analysis done in the BENEFIT study, ${ }^{32}$ which found that the risk for patients with CIS converting to clinically definite MS increased when either $\geq 9$ T2 lesions or gadolinium-positive MR imaging criteria were met. In the present study, neither of these conditions established any significant volumetric differences in the SDGM as well as in cortex between the CIS subgroups. This confirms the concept that there are pathogenetic mechanisms other than acute inflammation that may play a significant role in disease progression. ${ }^{33}$

We also investigated whether T2 lesion volume may be useful in subdividing the CIS cohort, as proposed in the past. ${ }^{34}$ The median T2 lesion volume was chosen because it, by definition, resulted in 2 groups with equal sizes. Patients with CIS with median T2 lesion volume $>4.49 \mathrm{~mL}$ showed lower total SDGM, caudate, thalamus, globus pallidus, hippocampus, and putamen volumes and higher NLVV, but not NCV, than those with median T2 lesion volume $<4.49 \mathrm{~mL}$. This finding further supports the concept that atrophy in the SDGM is relevant from the clinical onset of the disease and is consistent with results from previous studies. ${ }^{8,10,13}$ Hippocampal atrophy has been described in RRMS and progressive MS and has been related to both memory deficits and depressive symptoms. ${ }^{8,16,30,35,36}$ However, this is the first study to report evidence of hippocampal atrophy in patients with CIS. This finding is contrary to what has been previously reported by our own group ${ }^{8}$ and is probably related to the much larger sample size of patients with CIS in this study. Recent studies yielded similar results between different SDGM segmentation techniques used in the 2 studies, ${ }^{37,38}$ reducing the likelihood of the segmentation technique acting as a possible confounding factor.

We found evidence that the volumes of multiple SDGM

\begin{tabular}{|c|c|c|c|c|c|}
\hline & $\begin{array}{l}\text { CIS with T2-LV }<4.49 \mathrm{~mL} \\
(n=106)\left(\text { mean) }(\mathrm{SD})^{\mathrm{b}}\right.\end{array}$ & $\begin{array}{l}\text { CIS with T2-LV }>4.49 \mathrm{~mL} \\
(n=106)\left(\text { mean) }(\mathrm{SD})^{\mathrm{b}}\right.\end{array}$ & $\%$ Difference & SED & $P$ Value \\
\hline NBV & $1499(67.1)$ & $1487(80.1)$ & -0.8 & 6.5 & .238 \\
\hline NLVV & $35.3(9.8)$ & $40.3(12.4)$ & +14.2 & 0.5 & .001 \\
\hline NCV & $618.18(30.7)$ & $610.31(35.1)$ & -1.27 & 4.53 & .084 \\
\hline Total SDGM & $64.52(3.65)$ & $61.96(3.79)$ & -3.97 & 0.51 & $<.0001$ \\
\hline Caudate & $9.6(0.91)$ & $9.05(1.01)$ & -5.73 & 0.13 & $<.0001$ \\
\hline Putamen & $13.46(0.94)$ & $13.10(1.1)$ & -2.67 & 0.14 & .010 \\
\hline Globus pallidus & $4.8(0.37)$ & $4.68(0.34)$ & -2.50 & 0.04 & .007 \\
\hline Thalamus & $21.73(1.36)$ & $20.77(1.47)$ & -4.42 & 0.19 & $<.0001$ \\
\hline Hippocampus & $10.01(0.97)$ & $9.64(0.87)$ & -3.70 & 0.13 & .004 \\
\hline Amygdala & $3.59(0.41)$ & $3.45(0.46)$ & -3.90 & 0.06 & .021 \\
\hline Nucleus accumbens & $1.32(0.22)$ & $1.28(0.26)$ & -3.03 & 0.04 & .204 \\
\hline
\end{tabular}

Note:-SDGMV indicates subcortical deep gray matter volume; SED, standard error of difference; LV, lesion volume.

${ }^{a}$ All volumes are reported in milliliters.

${ }^{b}$ Student $t$ test was used to assess volumetric differences between patients with CIS with T2 lesion volume $<4.49 \mathrm{~mL}$ and those with CIS with $>4.49 \mathrm{~mL}$ T2 lesion volume. 


\begin{tabular}{|c|c|c|c|c|c|}
\hline & $\begin{array}{l}\mathrm{T} 2 \mathrm{LV} \\
r(P)\end{array}$ & $\begin{array}{l}\text { NBV } \\
r(P)\end{array}$ & $\begin{array}{l}\mathrm{NLVV} \\
\mathrm{r}(P)\end{array}$ & $\begin{array}{l}\text { EDSS } \\
r(P)\end{array}$ & $\begin{array}{c}\text { Disease Duratior } \\
r(P)\end{array}$ \\
\hline \multicolumn{6}{|l|}{ CIS $(n=212)$} \\
\hline NCV & $-.102(.140)$ & $.936(<.0001)^{\mathrm{a}}$ & $-.359(<.0001)^{a}$ & $-.149(.033)$ & $.035(.614)$ \\
\hline Total SDGMV & $-.329(<.0001)^{\mathrm{a}}$ & $.498(<.0001)^{a}$ & $-.178(.009)^{a}$ & $-.040(.568)$ & $-.126(.069)$ \\
\hline Caudate & $-.263(<.0001)^{\mathrm{a}}$ & $.430(<.0001)^{a}$ & $-.293(<.0001)^{\mathrm{a}}$ & $-.137(.051)$ & $-.071(.310)$ \\
\hline Putamen & $-.162(.018)$ & $.470(<.0001)^{\mathrm{a}}$ & $-.114(.098)$ & $-.049(.488)$ & $-.128(.065)$ \\
\hline Globus pallidus & $-.145(.035)$ & $.190(<.005)^{\mathrm{a}}$ & $.176(.010)$ & $-.063(.370)$ & $-.082(.239)$ \\
\hline Thalamus & $-.328(<.0001)^{a}$ & $.465(<.0001)^{\mathrm{a}}$ & $-.264(<.0001)^{\mathrm{a}}$ & $-.024(.734)$ & $-.033(.634)$ \\
\hline Hippocampus & $-.218(.001)^{\mathrm{a}}$ & $.162(.018)$ & $-.080(.244)$ & $.050(.476)$ & $-.095(.171)$ \\
\hline Amygdala & $-.132(.054)$ & $.074(.287)$ & $.092(.181)$ & $.059(.403)$ & $-.194(.005)^{\mathrm{a}}$ \\
\hline Nucleus accumbens & $-.132(.055)$ & $.370(<.0001)^{\mathrm{a}}$ & $-.141(.041)$ & $.065(.356)$ & $-.111(.110)$ \\
\hline \multicolumn{6}{|l|}{ Early RRMS ( $n=177)$} \\
\hline NCV & $-.420(<.0001)^{\mathrm{a}}$ & $.954(<.0001)^{\mathrm{a}}$ & $-.509(<.0001)^{a}$ & $-.204(.006)^{\mathrm{a}}$ & $-.383(<.0001)^{a}$ \\
\hline Total SDGMV & $-.613(<.0001)^{\mathrm{a}}$ & $.698(<.0001)^{\mathrm{a}}$ & $-.438(<.0001)^{\mathrm{a}}$ & $-.184(.014)$ & $-.322(<.0001)^{\mathrm{a}}$ \\
\hline Caudate & $-.558(<.0001)^{\mathrm{a}}$ & $.530(<.0001)^{\mathrm{a}}$ & $-.315(<.0001)^{\mathrm{a}}$ & $-.171(.023)$ & $-.282(<.0001)^{\mathrm{a}}$ \\
\hline Putamen & $-.520(<.0001)^{\mathrm{a}}$ & $.629(<.0001)^{\mathrm{a}}$ & $-.358(<.0001)^{\mathrm{a}}$ & $-.125(.098)$ & $-.306(<.0001)^{\mathrm{a}}$ \\
\hline Globus pallidus & $-.408(<.0001)^{\mathrm{a}}$ & $.342(<.0001)^{a}$ & $-.092(.223)$ & $-.070(.351)$ & $-.169(.024)$ \\
\hline Thalamus & $-.619(<.0001)^{\mathrm{a}}$ & $.721(<.0001)^{\mathrm{a}}$ & $-.604(<.0001)^{a}$ & $-.204(.007)^{\mathrm{a}}$ & $-.338(<.0001)^{a}$ \\
\hline Hippocampus & $-.312(<.0001)^{\mathrm{a}}$ & $.412(<.0001)^{a}$ & $-.140(.063)$ & $-.093(.216)$ & $-.173(.021)$ \\
\hline Amygdala & $-.246(.001)^{\mathrm{a}}$ & $.108(.152)$ & $-.017(.818)$ & $-.067(.375)$ & $-.124(.100)$ \\
\hline Nucleus accumbens & $-.335(<.0001)^{\mathrm{a}}$ & $.538(<.0001)^{\mathrm{a}}$ & $-.388(<.0001)^{\mathrm{a}}$ & $-.095(.208)$ & $-.164(.029)$ \\
\hline
\end{tabular}

Note:-LV indicates lesion volume; SDGMV, subcortical deep gray matter volume.

a Significant $P$ values $(P<.01)$.

structures were related to lesion and other atrophy outcomes in patients with CIS. In patients with early RRMS, these relationships became strong and were detected in all of the examined structures. While it is likely that T2 lesions play a role in subsequent SDGM atrophy development, the extent of that role cannot be established from the current data. Use of more specific MR imaging sequences like double inversion recovery can increase the ability to capture part of lesion GM pathology in vivo. ${ }^{4}$ It is highly likely that the development of SDGM and cortical atrophy is also related to other mechanisms different from inflammation, such as iron deposition. ${ }^{39,40}$ Similar observations were noticed when exploring the relationship between SDGM and cortical atrophy and clinical outcomes in this study. No relationship with disability was observed in the patients with CIS. Except for a weak correlation with the amygdala, no other structure was related to disease duration. On the other hand, a number of different SDGM structures as well as cortical volume were related to disease duration and EDSS in early RRMS.

While the study groups differed in SDGM but not cortical volumes, the exact relationship between atrophy in these regions remains unknown. The results suggest that, at least in the early stages of the disease, independent processes affect superficial and subcortical deep GM. The lack of a significant relationship between cortical atrophy and increased T2 lesion volume but modest-to-robust association with SDGM atrophy in patients with CIS indicates that SDGM atrophy is more closely associated with inflammation than cortical atrophy. Moreover, the association results from patients with early RRMS further support these findings.

The cross-sectional nature of this study limited our ability to monitor the evolution of SDGM atrophy on an individual basis. To address this shortcoming, longitudinal analyses of the SET and ASA studies at 2 and 5 years will be available in the near future. Using a regional T2 lesion volume-versus-SDGM and cortical atrophy approach with these datasets should help to further establish whether SDGM and cortical atrophy are a primary or secondary effect, or some combination of the $2 .{ }^{41}$ This study also did not use nonconventional MR imaging techniques that may provide additional insight into GM pathology. ${ }^{2}$

\section{Conclusions}

Our data suggest that significant SDGM atrophy but not cortical atrophy is evident from the earliest stages of the disease, strengthening the hypothesis that neurodegeneration and inflammation are both relevant in the conversion to clinically definite MS and disease progression.

\section{Acknowledgments}

We thank Eve Salczynski for technical assistance in the preparation of this manuscript and the subjects who participated in these studies.

Disclosures: Robert Zivadinov—RELATED: Grant. Biogen Idec; ${ }^{*}$ UNRELATED: Consultancy Teva Neuroscience, ${ }^{*}$ Biogen Idec, ${ }^{*}$ EMD Serono, ${ }^{*}$ Questcor Pharmaceuticals, ${ }^{*}$ Grants/ Grants Pending: Biogen Idec, Teva Neuroscience, Genzyme, Bracco, Questcor Pharmaceuticals, EMD Serono. Dana Horakova—RELATED: Grant. The ASA and the SET projects are supported by the Czech Ministry of Education, Research Program MSM 0021620849,* Support for Travel to Meetings for the Study or Other Purposes: Biogen Idec, Other. The ASA and the SET studies are investigator-initiated studies supported by Biogen Idec, UNRELATED: Travel/Accommodations/Meeting Expenses Unrelated to Activities Listed: Biogen Idec, Merck Serono, Bayer Shering. Ondrej Dolezal—RELATED: Grant. Biogen Idec, Support for Travel to Meetings for the Study or Other Purposes: Biogen Idec, UNRELATED: Consultancy. Biogen Idec, Payment for Lectures (including service on Speakers Bureaus). Novartis, Travel/Accommodations/Meeting Expenses Unrelated to Activities Listed: Merck Serono. Zdenek Seidl-RELATED: Grant. The ASA and the SET projects are supported by the Czech Ministry of Education, Research Program MSM 0021620849, Other. The ASA and the SET studies are investigator-initiated studies supported by Biogen Idec. Manuela Vaneckova-RELATED: Grant. The ASA and the SET projects are supported by the Czech Ministry of Education, Research Program MSM 0021620849, Other. The ASA and the SET 
studies are investigator-initiated studies supported by Biogen Idec. Jan KrasenskyRELATED: Grant. The ASA and the SET projects are supported by the Czech Ministry of Education, Research Program MSM 0021620849, Support for Travel to Meetings for the Study or Other Purposes: Biogen Idec, Comments: Research activities, Other. The ASA and the SET studies are investigator-initiated studies supported by Biogen Idec. Eva Havrdova-RELATED: Grant: Biogen Idec, ${ }^{*}$ Comments: Support for the MRI part of the study, UNRELATED: Board Membership: Biogen Idec, Merck, Teva, Novartis, Consultancy. Genzyme, Bayer, Grants/Grants Pending. Biogen Idec,* Novartis, ${ }^{*}$ Payment for Lectures (including service on Speakers Bureaus): Biogen Idec, Novartis, Payment for Development of Educational Presentations: Novartis, * Money paid to the institution.

\section{References}

1. Pirko I, Lucchinetti CF, Sriram S, et al. Gray matter involvement in multiple sclerosis. Neurology 2007;68:634-42

2. Poloni G, Minagar A, Haacke EM, et al. Recent developments in imaging of multiple sclerosis. Neurologist 2011;17:185-204

3. Geurts JJ, Stys PK, Minagar A, et al. Gray matter pathology in (chronic) MS: modern views on an early observation. J Neurol Sci 2009;282:12-20

4. Geurts JJ, Pouwels PJ, Uitdehaag BM, et al. Intracortical lesions in multiple sclerosis: improved detection with 3D double inversion-recovery MR imaging. Radiology 2005;236:254-60

5. Fisniku LK, Chard DT, Jackson JS, et al. Gray matter atrophy is related to long-term disability in multiple sclerosis. Ann Neurol 2008;64:247-54

6. Benedict R, Bruce J, Dwyer M, et al. Neocortical atrophy, third ventricular width, and cognitive dysfunction in multiple sclerosis. Arch Neurol 2006;63:1301-06

7. Fisher E, Lee JC, Nakamura K, et al. Gray matter atrophy in multiple sclerosis: a longitudinal study. Ann Neurol 2008;64:255-65

8. Ramasamy DP, Benedict RH, Cox JL, et al. Extent of cerebellum, subcortical and cortical atrophy in patients with MS: a case-control study. J Neurol Sci 2009;282:47-54

9. Audoin B, Ibarrola D, Malikova I, et al. Onset and underpinnings of white matter atrophy at the very early stage of multiple sclerosis: a two-year longitudinal MRI/MRSI study of corpus callosum. Mult Scler 2007;13:41-51

10. Audoin B, Zaaraoui W, Reuter F, et al. Atrophy mainly affects the limbic system and the deep grey matter at the first stage of multiple sclerosis. J Neurol Neurosurg Psychiatry 2010;81:690-95

11. Calabrese M, Atzori M, Bernardi V, et al. Cortical atrophy is relevant in multiple sclerosis at clinical onset. J Neurol 2007;254:1212-20

12. Fisniku LK, Altmann DR, Cercignani M, et al. Magnetization transfer ratio abnormalities reflect clinically relevant grey matter damage in multiple sclerosis. Mult Scler 2009;15:668-77

13. Henry RG, Shieh M, Amirbekian B, et al. Connecting white matter injury and thalamic atrophy in clinically isolated syndromes. J Neurol Sci 2009;282:61-66

14. Henry RG, Shieh M, Okuda DT, et al. Regional grey matter atrophy in clinically isolated syndromes at presentation. J Neurol Neurosurg Psychiatry 2008;79:1236-44

15. Raz E, Cercignani M, Sbardella E, et al. Clinically isolated syndrome suggestive of multiple sclerosis: voxelwise regional investigation of white and gray matter. Radiology 2010;254:227-34

16. Roosendaal SD, Bendfeldt $\mathrm{K}$, Vrenken $\mathrm{H}$, et al. Grey matter volume in a large cohort of MS patients: relation to MRI parameters and disability. Mult Scler 2011;17:1098-106

17. Calabrese M, Rinaldi F, Mattisi I, et al. The predictive value of gray matter atrophy in clinically isolated syndromes. Neurology 2011;77:257-63

18. Dalton CM, Chard DT, Davies GR, et al. Early development of multiple sclerosis is associated with progressive grey matter atrophy in patients presenting with clinically isolated syndromes. Brain 2004;127:1101-07

19. Raz E, Cercignani M, Sbardella E, et al. Gray- and white-matter changes 1 year after first clinical episode of multiple sclerosis: MR imaging. Radiology 2010;257:448-54

20. Rocca MA, Agosta F, Sormani MP, et al. A three-year, multi-parametric MRI study in patients at presentation with CIS. J Neurol 2008;255:683-91

21. Ceccarelli A, Rocca MA, Pagani E, et al. A voxel-based morphometry study of grey matter loss in MS patients with different clinical phenotypes. Neuroimage 2008;42:315-22

22. Havrdova E, Zivadinov R, Krasensky J, et al. Randomized study of interferon beta-1a, low-dose azathioprine, and low-dose corticosteroids in multiple sclerosis. Mult Scler 2009;15:965-76

23. Horakova D, Zivadinov R, Weinstock-Guttman B, et al. HLA DRB1*1501 is only modestly associated with lesion burden at the first demyelinating event. J Neuroimmunol 2011;236:76-80

24. Poser CM, Paty DW, Scheinberg L, et al. New diagnostic criteria for multiple sclerosis: guidelines for research protocols. Ann Neurol 1983;13:227-31

25. Zivadinov R, Rudick RA, De Masi R, et al. Effects of IV methylprednisolone on brain atrophy in relapsing-remitting MS. Neurology 2001;57:1239-47

26. Gelineau-Morel R, Tomassini V, Jenkinson M, et al. The effect of hypointense white matter lesions on automated gray matter segmentation in multiple sclerosis. Hum Brain Mapp 2011 Oct 5. [Epub ahead of print]

27. Yeh EA, Weinstock-Guttman B, Ramanathan M, et al. Magnetic resonance imaging characteristics of children and adults with paediatric-onset multiple sclerosis. Brain 2009;132:3392-400

28. Patenaude B, Smith S, Kennedy D, et al. A Bayesian model of shape and appearance for subcortical brain segmentation. Neuroimage 201;56:907-22

29. Batista S, Zivadinov R, Hoogs M, et al. Basal ganglia, thalamus and neocortical atrophy predicting slowed cognitive processing in multiple sclerosis. J Neuro 2012;259:139-46

30. Sicotte NL, Kern KC, Giesser BS, et al. Regional hippocampal atrophy in multiple sclerosis. Brain 2008;131:1134-41

31. Houtchens MK, Benedict RH, Killiany R, et al. Thalamic atrophy and cognition in multiple sclerosis. Neurology 2007;69:1213-23

32. Polman C, Kappos L, Freedman MS, et al. Subgroups of the BENEFIT study: risk of developing MS and treatment effect of interferon beta-1b. J Neurol 2008; $255: 480-87$

33. Gold R, Wolinsky JS, Amato MP, et al. Evolving expectations around early management of multiple sclerosis. Ther Adv Neurol Disord 2010;3:351-67

34. Filippi M, Horsfield MA, Morrissey SP, et al. Quantitative brain MRI lesion load predicts the course of clinically isolated syndromes suggestive of multiple sclerosis. Neurology 1994;44:635-41

35. Benedict RH, Ramasamy D, Munschauer F, et al. Memory impairment in multiple sclerosis: correlation with deep grey matter and mesial temporal atrophy. J Neurol Neurosurg Psychiatry 2009;80:201-06

36. Kiy G, Lehmann P, Hahn HK, et al. Decreased hippocampal volume, indirectly measured, is associated with depressive symptoms and consolidation deficits in multiple sclerosis. Mult Scler 2011;17:1088-97

37. Derakhshan M, Caramanos Z, Giacomini PS, et al. Evaluation of automated techniques for the quantification of grey matter atrophy in patients with multiple sclerosis. Neuroimage 2010;52:1261-67

38. Morey RA, Petty CM, Xu Y, et al. A comparison of automated segmentation and manual tracing for quantifying hippocampal and amygdala volumes. Neuroimage 2009;45:855-66

39. Khalil M, Enzinger C, Langkammer C, et al. Quantitative assessment of brain iron by $\mathrm{R}(2)^{*}$ relaxometry in patients with clinically isolated syndrome and relapsing-remitting multiple sclerosis. Mult Scler 2009;15:1048-54

40. Ceccarelli A, Rocca MA, Neema M, et al. Deep gray matter T2 hypointensity is present in patients with clinically isolated syndromes suggestive of multiple sclerosis. Mult Scler 2009;16:39-44

41. Antulov R, Carone DA, Bruce J, et al. Regionally distinct white matter lesions do not contribute to regional gray matter atrophy in patients with multiple sclerosis. J Neuroimaging 2011;21:210-18 